the identification of MACE with no loss of sensitivity and excellent negative predictive value. (100\% sens, $86 \%$ spec, $100 \%$ NPV).

Conclusion The CENTAUR hs-TnI assay and HEART score with modifications would facilitate the early discharge of patients with chest pain from ED within 4 hours without missing any MACE at 30 days.

Conflict of Interest This is part of the finding of my thesis for MSc which will need to be completed in September 2019.

\section{CHARACTERIZATION AND OUTCOMES OF SPONTANEOUS CORONARY ARTERY DISSECTION PATIENTS PRESENTING WITH AN ACUTE CORONARY SYNDROME: INSIGHTS FROM THE ACALM REGISTRY}

${ }^{1}$ Rahul Potluri*, ${ }^{2}$ Paul Carter, ${ }^{3}$ Amir Aziz, ${ }^{4}$ Minh Vo, ${ }^{5}$ Ranjit More, ${ }^{6}$ Jaydeep Sarma, ${ }^{7}$ Asif Ahmed, ${ }^{8}$ David Adlam, ${ }^{4}$ Robert Welsh, ${ }^{9}$ Kevin Bainey. ${ }^{1}$ Blackpool Teaching Hospitals; ${ }^{2}$ ACALM Study Unit; ${ }^{3}$ New Cross Hospital; ${ }^{4}$ Mazankowski Alberta Heart Institute, University of Alberta, Edmonton, Canada; ${ }^{5}$ Blackpool Teaching Hospital NHS Foundation Trust; ${ }^{6}$ Manchester University Foundation Trust; ${ }^{7}$ Aston Medical School, Aston University; ${ }^{8}$ Department of Cardiovascular Sciences, Glenfield Hospital; ${ }^{9}$ Mazankowski Alberta Heart Institute

\subsection{6/heartjnl-2019-BCS.161}

Introduction Spontaneous coronary artery dissection (SCAD) is recognised as an infrequent presentation of acute coronary syndromes (ACS). Using a large comprehensive UK ACS registry, we examined the incidence, demographic profile and longterm survival of ACS patients with SCAD.

Methods We compared ACS patients with SCAD versus without SCAD admitted to hospitals in the UK (England) between 2000 and 2014 from the Algorithm for Co-morbidity, Associations, Length of stay and Mortality (ACALM) registry $(n=33$, 163). ACALM uses a big data approach collecting ICD-10 and OPCS-4 coding systems to identify patient data. Primary outcome was all-cause mortality over a 14-year time period. Adjustment was performed using a Cox-regression model.

Results In our cohort, 182 patients (0.54\%) were diagnosed with SCAD as the cause for ACS. Compared to patients without SCAD, patients with SCAD were younger, more commonly female and less likely to have traditional risk factors for ACS or significant comorbid conditions (Table 1). Revascularization with percutaneous coronary intervention (PCI) or coronary artery bypass graft (CABG) surgery was uncommon with SCAD $(11.0 \%, 2.7 \%$ respectively) compared to ACS patients without SCAD $(50.7 \%, 10.6 \%$ respectively). All-cause mortality was significantly better in SCAD patients compared to non-SCAD ACS patients $(10.4 \%$ vs. $32.1 \%, \mathrm{p}=0.003)$. Kaplan-Meier curve comparing 5-year survival is shown below (figure 1). After adjusting for age, gender, ethnic group and the top causes of morbidity and mortality in the UK, patients with SCAD presenting as ACS demonstrate improved survival compared to ACS patients without SCAD (OR 1.89; 95\% C.I 1.20-2.96).

Conclusion Accepting the potential of under reporting by clinicians in routine clinical practice, a minority of ACS patients in this large UK registry present with SCAD. These patients are commonly younger females without traditional risk factors for ACS. To our knowledge, this is the first study to demonstrate improved long-term survival over a 14 -year period with SCAD. Additionally, our analysis suggests conservative therapy is safe in this patient population. Further studies are required
Abstract 164 Table 1 Demographics, management and outcomes of ACS patients \pm SCAD

\begin{tabular}{|l|l|l|l|}
\hline & No SCAD & SCAD & p value \\
\hline N & 32981 & 182 & \\
\hline Mean age +/-S.D, years & $66.2+/-14.3$ & $51.9+/-20.4$ & $<0.01$ \\
\hline Female Gender (\%) & $11546(35.3)$ & $125(68.7)$ & $<0.01$ \\
\hline Risk Factors and comorbid conditions\% & & & \\
Hypertension & 47.6 & 38.5 & 0.04 \\
Type 2 Diabetes Mellitus & 20.7 & 11.5 & 0.02 \\
Hyperlipidaemia & 26.2 & 21.4 & 0.45 \\
PVD & 4.8 & 6.6 & 0.03 \\
Ischaemic Stroke & 4.5 & 0.5 & 0.03 \\
Heart Failure & 19.1 & 8.2 & $<0.01$ \\
Atrial Fibrillation & 13.7 & 10.4 & 0.54 \\
Chronic Kidney Disease & 6.3 & 4.9 & 0.83 \\
Anaemia & 5.9 & 5.2 & 0.45 \\
Cardiac Arrest & 2.3 & 1.1 & 0.35 \\
Obesity & 2.0 & 2.2 & 0.44 \\
Cardiogenic Shock & 1.0 & 0.0 & 0.99 \\
Cocaine abuse & 0.1 & 0.5 & $\mathbf{0 . 0 3}$ \\
\hline Management & & & \\
Revascularisation \% & & & \\
Percutaneous Coronary Intervention (PCI) & 50.7 & 10.6 & $<0.01$ \\
Coronary artery bypass graft (CABG) & 11.0 & 2.7 & $<0.01$ \\
\hline Outcomes & & & \\
Mean follow-up length, days & 2186 & 2208 & 0.77 \\
Mortality over the follow-up period, \% & 32.1 & 10.4 & $<0.01$ \\
Mean readmissions to hospital & 9.6 & 7.6 & $\mathbf{0 . 0 2}$ \\
Subsequent heart failure, \% & 9.7 & 5.5 & 0.07 \\
Subsequent ACS, \% & 67.4 & 15.6 & $<0.01$ \\
Mean length of hospital stay of index admission, days & 6.5 & 7.2 & $\mathbf{0 . 0 3}$ \\
\hline
\end{tabular}

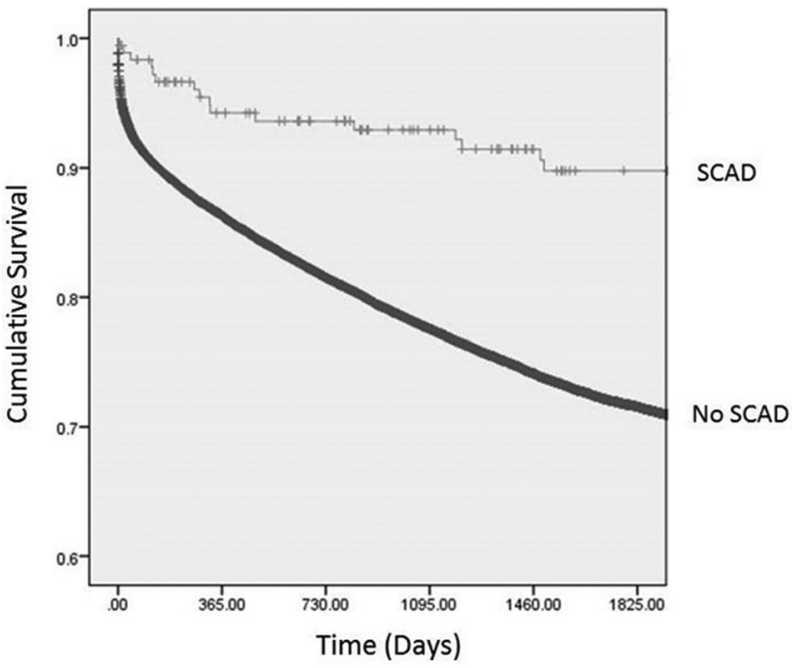

Abstract 164 Figure 1 Kaplan-Meier curve showing 5 year survival in ACS patients \pm SCAD

to explore non-traditional risk factors, predictors of SCAD and best treatment strategy for these patients.

Conflict of Interest Nil

\section{FRAIL OLDER PATIENTS HAVE A GREATER IMPROVEMENT IN PHYSICAL QUALITY OF LIFE FOLLOWING INVASIVE MANAGEMENT FOR NON-ST ELEVATION ACUTE CORONARY SYNDROME}

${ }^{1}$ Zhaotao Sophie Gu*, ${ }^{2}$ Danny Chan, ${ }^{3}$ Ben Beska, ${ }^{4}$ Hannah Sinclair, ${ }^{5}$ Weiliang Qiu,

${ }^{6}$ Vijay Kunadian. ${ }^{1}$ Newcastle University; ${ }^{2}$ Freeman Hospital; ${ }^{3}$ Harvard University

\subsection{6/heartjnl-2019-BCS.162}

Introduction Frailty, defined as a vulnerability to physiological stressors due to a decline in reserve with age, is common in older patients with non-ST elevation acute coronary syndrome 